\title{
Update Mammakarzinom 2018 (Teil 3) - Genomforschung, individualisierte Medizin und Immuntherapien - mitten in einer neuen Ära: Prävention und Therapie des frühen Mammakarzinoms
}

\author{
Update Breast Cancer 2018 (Part 3) - Genomics, Individualized \\ Medicine and Immune Therapies - in the Middle of a New Era: \\ Prevention and Treatment Strategies for Early Breast Cancer
}

\section{(ㄷ) (i) (오 $\Theta$}

\section{Autoren}

Achim Wöckel ${ }^{1}$, Michael P. Lux ${ }^{2}$, Wolfgang Janni ${ }^{3}$, Andreas D. Hartkopf ${ }^{4}$, Naiba Nabieva ${ }^{2}$, Florin-Andrei Taran ${ }^{4}$, Friedrich Overkamp ${ }^{5}$, Peyman Hadji ${ }^{6}$, Hans Tesch ${ }^{7}$, Johannes Ettl ${ }^{8}$, Diana Lüftner ${ }^{9}$, Volkmar Müller $^{10}$, Manfred Welslau ${ }^{11}$, Erik Belleville ${ }^{12}$, Sara Y. Brucker ${ }^{4}$, Florian Schütz ${ }^{13}$, Peter A. Fasching ${ }^{2}$, Tanja N. Fehm ${ }^{14}$, Andreas Schneeweiss ${ }^{13,15}$, Hans-Christian Kolberg ${ }^{16}$

Institute

1 Department of Gynecology and Obstetrics, University Hospital Würzburg

2 Erlangen University Hospital, Department of Gynecology and Obstetrics, Comprehensive Cancer Center ErlangenEMN, Friedrich-Alexander University Erlangen-Nuremberg, Erlangen

3 Department of Gynecology and Obstetrics, Ulm University Hospital, Ulm

4 Department of Obstetrics and Gynecology, University of Tübingen, Tübingen

5 OncoConsult Hamburg GmbH, Hamburg

6 Department of Bone Oncology, Nordwest Hospital, Frankfurt

7 Oncology Practice at Bethanien Hospital Frankfurt, Frankfurt

8 Department of Obstetrics and Gynecology, Klinikum rechts der Isar, Technical University of Munich, Munich

9 Charité University Hospital, Berlin, Campus Benjamin

Franklin, Department of Hematology, Oncology and Tumour Immunology, Berlin

10 Department of Gynecology, Hamburg-Eppendorf University Medical Center, Hamburg

11 Onkologie Aschaffenburg, Hämatolo-Onkologische Schwerpunktpraxis am Klinikum Aschaffenburg, Aschaffenburg

12 ClinSol GmbH \& Co KG, Würzburg

13 Department of Obstetrics and Gynecology, University of Heidelberg, Heidelberg

14 Department of Gynecology and Obstetrics, University Hospital Düsseldorf, Düsseldorf

15 National Center for Tumor Diseases, Division Gynecologic Oncology, University Hospital Heidelberg, Heidelberg

16 Department of Gynecology and Obstetrics, Marienhospital Bottrop, Bottrop
Schlüsselwörter

Mammakarzinom, Behandlung, Metastasen, CDK4/6, PD1/

PDL1, Studien, Risiko, Prävention

Key words

breast cancer, treatment, metastases, CDK4/6, PD1/PDL1,

studies, risk, prevention

eingereicht 9.8.2018

akzeptiert 23.8.2018

Bibliografie

DOI https://doi.org/10.1055/a-0715-2821

Geburtsh Frauenheilk 2018; 78: 1110-1119 @ Georg Thieme

Verlag KG Stuttgart · New York I ISSN 0016-5751

Korrespondenzadresse

Peter A. Fasching, MD

Erlangen University Hospital, Department of Gynecology and Obstetrics, Comprehensive Cancer Center Erlangen EMN,

Friedrich Alexander University of Erlangen-Nuremberg

Universitätsstraße 21-23, 91054 Erlangen

peter.fasching@uk-erlangen.de

\section{ZUSAMMENFASSUNG}

Beim primären, frühen Mammakarzinom zielt die Behandlungsplanung auf ein immer besseres Verständnis der Erkrankung ab. Die Identifikation von Patientinnen mit einer exzellenten Prognose könnte dieser Gruppe helfen, unnötige Therapien zu vermeiden. Weiterhin wird die Planung der Therapie immer weiter auf die Patientin abgestimmt. Das Wissen über Patientinnen, die besonders von einer Chemotherapie profitieren, wächst genauso wie das Wissen um Patientinnen, 
die von einer Immuntherapie profitieren könnten. Hinsichtlich der Immuntherapien stehen die durchgeführten Studien kurz vor der Publikation. Einzelne kleinere Studien bieten einen ersten Einblick in die Wirksamkeit der Checkpoint-Inhibitoren (Anti-PD1/PDL1-Therapien). Nicht zuletzt konnte kürzlich eine der größten Brustkrebsstudien aller Zeiten zu Ende geführt werden. Die Anwendung eines Multigentests konnte zeigen, dass er ausreicht, um Patientinnen mit einer so guten Prognose zu identifizieren, dass keine Chemotherapie nötig ist. Dieser Review-Artikel soll die aktuellen Studien zusammenfassen und einen Ausblick der gegenwärtigen Entwicklungen geben.

\section{ABSTRACT}

In primary early breast cancer, the aim of treatment planning is to obtain an increasingly better understanding of the disease. The identification of patients with an excellent progno- sis could help this group avoid unnecessary treatments. Furthermore, the planning of treatment is becoming increasingly patient-focussed. There is a growing understanding of those patients who benefit particularly from chemotherapy, as well as of those who could benefit from immunotherapy. Studies conducted on immunotherapies will be published shortly. Smaller individual studies offer an initial insight into the efficacy of checkpoint inhibitors (anti-PD1/PDL1 therapies). Not least, one of the largest breast cancer studies of all times has recently come to an end. The use of a multigene test has shown that it is sufficient to identify patients with such a good prognosis that chemotherapy is unnecessary. This review article is intended to summarise the current studies and give an outlook on current developments.

\section{Einführung}

Eines der Grundprinzipien des medizinischen Handelns, „primum non nocere“, lateinisch für „erstens nicht schaden“, ist im Jahr 2018 so aktuell wie je zuvor. Dies gilt insbesondere bei den Krebskrankheiten. Auch die weiteren Punkte dieser hippokratischen Tradition wie „zweitens vorsichtig sein“ und „drittens heilen“ finden ihre Korrelate in den aktuellen Studienergebnissen, den Leitlinien und Therapieempfehlungen [1 - 3]. Für Karzinome bedeutet dies, dass die Identifikation von Patientinnen mit einer guten und schlechten Prognose, mit einem guten und schlechten Therapieansprechen und schweren vs. leichten Nebenwirkungen nach wie vor eines der Hauptforschungsgebiete und Translationsbestrebungen für die klinische Praxis ist. Mehr denn je gehen die aktuellen Entwicklungen in diese Richtung, sodass in den letzten Monaten auf großen internationalen Kongressen wie dem der American Society of Oncology 2018, der American Association of Cancer Research 2018, dem Kongress der Deutschen Gesellschaft für Senologie und dem Krebskongress 2018 sowie in internationalen Publikationen eine Reihe von Studien veröffentlicht wurden, die in dieser Übersichtsarbeit zusammengefasst werden sollen.

\section{Prävention}

\section{Individualisierte Risikoprädiktion}

Nach wie vor ist nur ein Teil der Mammakarzinome durch Risikofaktoren erklärbar. In den letzten Jahren ist insbesondere an den genetischen Risikofaktoren und der mammografischen Dichte gearbeitet worden. Im Bereich der genetischen Risikofaktoren wurde in den letzten 10 Jahren eine enorme Menge an Daten generiert, die insgesamt ca. 38\% eines 2-fach erhöhten familiären relativen Risikos erklären [4-7]. Hiervon werden ca. 20\% durch moderat- bis hoch-penetrante Gene (BRCA1, BRCA2, CHEK2, PALB2 etc.) erklärt und $18 \%$ durch häufige Risikovarianten, von denen ca. 173 validiert und veröffentlich sind [4-30]. Außerdem wird geschätzt, dass weitere $23 \%$ des 2 -fach erhöhten, familiären rela- tiven Risikos durch häufige Varianten erklärt werden können, die noch nicht beschrieben sind [7]. Dieses Wissen wird genutzt, um sogenannte polygenische Risikoscores oder Prädiktionsmodelle für das individuelle Brustkrebsrisiko oder spezifische Subtypen zu entwickeln [31-36], welche dann in individualisierte Früherkennungsmaßnahmen umgesetzt werden können, wenn auch bislang nur in Studien [32, 37-41]. Nicht nur in der Risikoprädiktion, sondern auch bei der Behandlung scheinen Keimbahnvarianten immer mehr eine Rolle zu spielen für die Therapiewahl und Prädiktion der Therapieeffektivität [42-46].

\section{Neue medikamentöse Präventionsstrategien}

In Bezug auf eine tatsächliche primäre Prävention konnte kürzlich gezeigt werden, dass bei BRCA1-Mutationsträgern der RANKL/ RANK/OPG-Pathway eine besondere Rolle spielt [47-50]. Basierend auf Voruntersuchungen, die u.a. eine Kohorte von BRCA1Mutationsträgerinnen beinhaltete, die nach einer Therapie mit Denosumab mit einer Reduktion der Proliferation von Brustepithelzellen reagierten [47], wird nun eine Präventionsstudie für BRCA1-Mutationsträgerinnen mit Denosumab durchgeführt [51]. Diese Therapie hätte mit einem akzeptablen Nebenwirkungsprofil für diese individuelle Risikopopulation bei einer Wirksamkeit ein gutes Nutzen-Risiko-Profil.

\section{Neue lokale, radiotherapeutische Präventions- strategien}

Für Patientinnen mit Mammakarzinom und einer BRCA1/2-Mutation stellt sich auch die Frage, wie mit dem Risiko für ein kontralaterales Mammakarzinom umgegangen wird. Das kumulative Risiko in den ersten 20 Jahren nach einer Mammakarzinomerkrankung beträgt ca. 40\% [52]. Für diese Patientinnen wird die Möglichkeit einer kontralateralen Mastektomie diskutiert [53, 54]. Eine weitere experimentelle Option, die kontralaterale Bestrahlung, wurde in einer kürzlich vorgestellten Studie untersucht [55]. In dieser Phase-II-Studie wurden 162 Patientinnen mit BRCA1/2-Mutation eingeschlossen, von denen sich 81 für und 81 gegen eine Bestrahlung der kontralateralen Brust entschieden hatten [55]. 
Nach einem medianen Follow-up von 60 Monaten hatten 9 Patientinnen in der nicht bestrahlten Gruppe ein kontralaterales Mammakarzinom entwickelt, während dies nur bei 2 Patientinnen in der bestrahlten Gruppe der Fall war $(p=0,027)$. Interessanterweise waren die 9 Rezidive in der nicht bestrahlten Gruppe nach im Median 24 Monaten aufgetreten, während die 2 Rezidive in der bestrahlten Gruppe nach 80 und 109 Monaten auftraten [55]. Es traten weder Früh- noch Spätkomplikationen der Bestrahlung in der bestrahlten Gruppe auf, sodass die kontralaterale Radiatio für Patientinnen mit BRCA1/2-Mutation eine Alternative zur prophylaktischen kontralateralen Mastektomie darstellen könnte.

\section{Das frühe Mammakarzinom - Lokaltherapie}

Die Lokaltherapie, also die Entfernung aller Tumorzellen aus der Brust und den lokoregionären Lymphknoten, stellt die Grundlage der primären Behandlung des Mammakarzinoms dar. Einige Aspekte der Bestrahlung der lokoregionären Lymphknoten werden weiterhin untersucht. Die EORTC-22922/10925-Studie untersuchte den Wert der Bestrahlung der medialen supraklavikulären Lymphknoten sowie der Mammaria-interna-Lymphknoten beim Mammakarzinom in den klinischen Stadien I-III [56]. Nun sind die Auswertungen des 15-Jahres-Follow-up präsentiert worden [57]. In die Studie waren 4004 Patientinnen mit befallenen axillären Lymphknoten und/oder medialem Tumorsitz eingeschlossen worden. Die Patientinnen wurden für oder gegen die Bestrahlung der oben genannten Lymphknotenstationen randomisiert. Etwas über die Hälfte der Patientinnen (55,6\%) wurden mit befallenen Lymphknoten eingeschlossen, während die übrigen Patientinnen zwar negative Lymphknoten, aber eine mediale Tumorlokalisation hatten. Primäres Studienziel war die Identifikation eines Unterschiedes von 4,0\% im 10-Jahres-Gesamtüberleben zwischen den beiden Armen, wobei dieses Studienziel mit einem knapp nicht signifikanten Benefit von 1,6\% ( $p=0,056)$ nicht erreicht wurde [56].

In der nun vorgestellten Analyse mit einem medianen Followup von 15,7 Jahren zeigte sich zwar numerisch ein etwas größerer Benefit bezüglich des Gesamtüberlebens für die extensive Bestrahlung von $2,4 \%$, allerdings war dieser Unterschied weiter nicht signifikant $(p=0,358)$ [57]. Die Brustkrebssterblichkeit war zwar um $3,9 \%$ signifikant besser ( $p=0,005)$, ebenso wie die Rezidivrate um 2,6\% ( $p=0,024)$ [57], jedoch haben die Autoren zum jetzigen Zeitpunkt noch keine Erklärung dafür, warum dies sich nicht in einen signifikanten Gesamtüberlebensvorteil umsetzt [57]. Bevor die von den Autoren angekündigten extensiven Subgruppenanalysen nicht publiziert sind, lässt sich daher weder der Wert der intensiveren Bestrahlung insgesamt noch die Frage der besonders profitierenden Patientinnen endgültig beurteilen.

Dass die Strahlentherapie von Tumoren nicht nur eine lokale Wirkung hat, ist schon seit einiger Zeit bekannt [58]. Der Effekt, dass die Bestrahlung nicht nur lokales Ansprechen, sondern auch eine Wirkung an entfernten, nicht bestrahlten Regionen erreichen kann, wird als „abskopaler Effekt“ bezeichnet. Die dahinterliegenden Mechanismen sind nicht gut verstanden und der Effekt wird therapeutisch bislang nicht genutzt [58]. Da die vermuteten Ef- fekte immunologischer Natur sind, könnte in Kombination mit neuen, immunologischen Therapien versucht werden, diese Effekte für die Krebstherapie nutzbar zu machen.

Eine kürzlich präsentierte Phase-II-Studie zur Kombination von Bestrahlung und Pembrolizumab beim metastasierten triple-negativen Mammakarzinom zeigte hier vielversprechende Ergebnisse [59]. Auch wenn die Gruppe der nach 13 Wochen auswertbaren Patientinnen mit 9 klein war, zeigte doch die Ansprechrate von 33\% außerhalb der bestrahlten Lokalisationen mit einem bis zu 40 Wochen anhaltenden Ansprechen einen deutlichen Unterschied zu der in einer solchen Kohorte mit im Median 3 zytotoxischen Therapien vor Studieneinschluss zu erwartenden Ansprechrate von nur $5-7 \%$. Dies ist somit ein weiterer Hinweis auf die möglichen Effekte einer Kombination von Radiatio und Immuntherapie, zu der bereits vielversprechende Ergebnisse vorliegen $[60,61]$.

\section{Das frühe Mammakarzinom - die systemische Behandlung der prämenopausalen Patientin}

\section{Die Datenlage zu Aromatasehemmern wächst}

Der adjuvante Einsatz von Tamoxifen (TAM) bei prämenopausalen Frauen mit hormonrezeptorpositivem Mammakarzinom gehört zu den effektivsten Therapieoptionen [62]. Bei postmenopausalen Patientinnen sind Aromataseinhibitoren (Al) als die effektivste Behandlung einer der Standards [63]. Für prämenopausale Patientinnen wurden einige Studien durchgeführt, die Aromatasehemmer mit gleichzeitiger ovarieller Suppression untersucht haben [6466].

Eine kürzliche Auswertung der SOFT- und der TEXT-Studie zeigte nun, dass bei Frauen, die innerhalb von 8 Monaten nach Erhalt einer Chemotherapie weiterhin/wieder prämenopausal waren, der zusätzliche Einsatz einer ovariellen Suppression (OFS, z. B. durch Gabe eines GnRH-Analogon) erwogen werden kann. In einer aktuellen Auswertung der 8-Jahres-Daten aus den beiden Studien verbessert dieses Vorgehen das krankheitsfreie und gesamte Überleben signifikant [66]. Verwendet man anstelle von TAM einen Al, wird das Rezidivrisiko zusätzlich um 2-3\% reduziert, allerdings zeigt sich hier kein Einfluss auf das gesamte Überleben bei klinisch relevanter Zunahme der Morbidität [66].

Ebenso wurde kürzlich eine risikoadaptierte Analyse des fernrezidivfreien Überlebens von HER2-negativen Patientinnen aus den SOFT- und TEXT-Studien vorgestellt [67]. Es wurde für jede Patientin ein „Composite Recurrence Risk Index“ (CRRI) basierend auf Alter, Nodalstatus, Tumorgröße, Grading, ER-/und PR-Status sowie Ki-67-Expression berechnet. Während in der gesamten Population durch den Einsatz eines Al + OFS im Vergleich zu TAM + OFS das Fernrezidivrisiko um 3\% reduziert wurde, waren es bei Patientinnen mit hohem klinischen Risiko 15\% [67]. Tam + OFS verbesserte im Vergleich zu TAM alleine das fernrezidivfreie Überleben von Frauen mit hohem Risiko um 10\%. Bei Patientinnen, die lediglich TAM erhielten, traten höhergradige Nebenwirkungen in $25 \%$ der Fälle auf; in der TAM + OFS-Gruppe waren es $31 \%$ und in der Al+OFS-Gruppe 32\% der Frauen [67]. 
Eine ähnliche Fragestellung griffen Noh et al. in der ASTRRAStudie auf [68]. Hier wurde der Menopausenstatus von 1483 initial prämenopausalen Frauen unter 45 nach Abschluss einer adjuvanten oder neoadjuvanten Chemotherapie klinisch und laborchemisch nachverfolgt. Sicher prämenopausale Frauen erhielten 5 Jahre TAM vs. 5 Jahre TAM + 2 Jahre OFS. 24 Monate nach Abschluss der Chemotherapie waren $>90 \%$ aller Studienteilnehmerinnen (wieder) prämenopausal. Insgesamt wurden 1282 Patientinnen randomisiert. Nach einem medianen Follow-up von 63 Monaten waren $88 \%$ der Patientinnen, die lediglich TAM erhalten hatten, rezidivfrei. In der TAM + OFS-Gruppe waren es hingegen 91\% der Patientinnen ( $p=0.033)$.

Beide Analysen bestätigen die derzeitige Empfehlung der S3Leitlinie [69], dass die Kombination der endokrinen Therapie mit einer OFS nur bei prämenopausalen Frauen mit hohem Risiko und prämenopausaler Situation nach Chemotherapie erwogen werden kann, da, insbesondere in Hinblick auf Lebensqualität und Compliance, die gesteigerte Rate an Nebenwirkungen berücksichtigt werden muss. Im Hinblick auf die erhöhte Rate an Nebenwirkungen, den fehlenden Einfluss auf das gesamte Überleben und die nur geringe Reduktion des Rezidivrisikos sollte TAM ( \pm OFS) nur in Einzelfällen (z. B. bei Kontraindikationen) durch einen $\mathrm{Al}+$ OFS ersetzt werden. Die endokrine Therapie mit TAM bleibt daher die Standardbehandlung von prämenopausalen Frauen mit frühem Mammakarzinom.

\section{Das frühe Mammakarzinom - die systemische Behandlung mit Denosumab}

In Bezug auf den adjuvanten Einsatz der Bisphosphonate hat die Oxford-Metaanalyse zu einem Paradigmenwechsel in internationalen Leitlinien geführt [70]. Übereinstimmend mit vielen Einzelstudien hat diese eine 34\%ige Verringerung des relativen Risikos für das Auftreten von Knochenmetastasen sowie eine 17\%ige Mortalitätsreduktion bei Frauen mit hormonrezeptorpositivem Mammakarzinom unter hormonentziehender Therapie bzw. in der Postmenopause gezeigt und somit die brustkrebsspezifischen Vorteile einer „lediglichen“ Osteoporosetherapie belegt.

In Bezug auf den monoklonalen Antikörper Denosumab (Dmab) lag bislang nur eine Zwischenauswertung der ABCSG-18Studie vor [71]. Vor diesem Hintergrund hatte man sich von der Auswertung der D-CARE-Studie sowie der Ergebnisse der ABCSG18-Studie hierzu eine Klärung in Bezug auf den möglichen adjuvanten Einsatz von Dmab erwartet.

Coleman et al. untersuchten im Rahmen der D-CARE-Studie den adjuvanten Einfluss von Dmab vs. Placebo auf das knochenmetastasenfreie Überleben (BMFS) sowie diverse weitere sekundäre Endpunkte (DFS, OS) bei 4509 prä- sowie postmenopausalen Frauen mit hohem Rezidivrisiko [72]. Nach einer neoadjuvanten/ adjuvanten CHT erfolgte die Randomisierung < 12 Wochen postoperativ. Eingeschlossen wurden überwiegend Frauen mit einem hormonrezeptorpositiven, HER2-negativen, nodal-positiven Mammakarzinom, G 2-3. Über 95\% erhielten eine adjuvante CHT, über $90 \%$ der hormonrezeptorpositiven Patientinnen einen $\mathrm{Al}$ und 79\% der HER2-positiven Patientinnen eine entsprechende AntiHER2-Therapie. Hervorzuheben ist weiterhin das dosisdichte
Schema mit 6-monatlichen Gaben, gefolgt von einer quartalsweisen Applikation von $120 \mathrm{mg}$ Dmab vs. Placebo. Aufgrund der unerwartet niedrigen Rezidivrate erfolgte ein Amendment und ein Wechsel von einer „event-driven“ zu einer „time-driven“-Analyse. Die Ergebnisse zeigten in Bezug auf den primären sowie auf die sekundären Endpunkte keine signifikanten Unterschiede (BMFS, DFS, OS). In Bezug auf die Nebenwirkungen zeigten sich unter Dmab vs. Placebo 122 vs. 4 Kieferosteonekrosen (ONJ) sowie 9 vs. 0 atypische Femurfrakturen (AFF). Zusammenfassend hat sich bei dem untersuchten Kollektiv kein Vorteil einer adjuvanten Denosumab-Gabe nachweisen lassen bei einer insgesamt inakzeptabel hohen Nebenwirkungsrate.

Gnant et al. präsentierten die Ergebnisse der ABCSG-18-Studie nach einer medianen Beobachtungsdauer von 73 Monaten [73]. Untersucht wurde der Einfluss von $60 \mathrm{mg}$ Dmab (alle 6 Monate) vs. Placebo bei 3425 postmenopausalen, hormonrezeptorpositiven Frauen mit Mammakarzinom unter einer adjuvanten Al-Therapie mit insgesamt niedrigem Rezidivrisiko. Der primäre Endpunkt lag in der Zeit bis zur ersten klinischen, osteoporosebedingten Fraktur und wurde mit einer RR von 50\% eindrucksvoll und deutlich früher als erwartet erreicht. Aufgrund der unerwartet schnellen und deutlichen Risikoreduktion von Frakturen wurde auf Rat des IDMC/SC den eingeschlossenen Patientinnen die Möglichkeit zur Entblindung eingeräumt, um ggf. von Placebo auf Dmab für einen Zeitraum von 3 Jahren (Open-Label-Phase) wechseln zu können. Hiervon machten 278 Frauen Gebrauch, wobei 252 dieser Frauen von Placebo zu Dmab wechselten. Aus diesem Grund konnte der sekundäre Endpunkt DFS (OS und BMFS wurden nicht vorgestellt) nur deskriptiv ausgewertet werden. Das mittlere Alter der Studienpopulation lag bei 64 Jahren, > 70\% hatten ein invasiv duktales Mammakarzinom, T1-2, nodal-negativ, und wiesen G1-2-Tumoren auf. Alle Patientinnen waren hormonrezeptorpositiv, > 90\% HER-2-positiv, wobei > 75\% eine neo- bzw. adjuvante CHT erhielten.

Nach einer medianen Beobachtungsdauer von 73 Monaten zeigte sich in Bezug auf den sekundären Endpunkt DFS in einer deskriptiven Analyse eine Hazard Ratio von 0,82 (0,69-0,98), $p<0,025$ (Log Rank). Aufgrund der unerwartet hohen Frakturreduktion und der gewährten Option zum frühzeitigen Wechsel von Placebo auf Dmab erfolgte noch eine Reihe weiterer Analysen (z. B. unter Ausschluss der o.g. Frauen in der Cross-over-Gruppe), welche alle gleichsinnig die o.g. Risikoreduktion des DFS bestätigten. Dieses Ergebnis war am stärksten ausgeprägt, wenn weniger als 3 Monate zwischen Start der Al-Therapie und der Erstapplikation von Dmab lagen. Die Aufschlüsselung der DFS-Ereignisse ergab lediglich bei den nicht histologisch gesicherten Fernmetastasen (DFS) oder sekundären invasiven Karzinomen (kein Mammakarzinom) eine numerische Überlegenheit, während sich in Bezug auf Lokalrezidive, DCIS, kontralaterale Mammakarzinome und histologisch gesicherte Fernmetastasen (DFS) keine Unterschiede zwischen Dmab und Placebo zeigten. Bezüglich der Nebenwirkungen gab es keine signifikanten Gruppenunterschiede [73]. In der ABCSG-18 kam es zu keinem Fall einer Kieferosteonekrose (ONJ) oder atypischen Femurfrakturen (AFF). Zusammenfassend zeigte die ABCSG-18-Studie neben der bereits veröffentlichten signifikanten Reduktion von osteoporosebedingten klinischen Frakturen eine Verbesserung des DFS auf. Diese Überlegenheit war be- 
sonders in Bezug auf nicht histologisch gesicherte Fernmetastasen (DFS) sowie sekundäre invasive Karzinome (kein Mammakarzinom) nachweisbar.

Auf den ersten Blick scheinen diese Studien vollkommen gegensätzliche Ergebnisse aufzuzeigen. Es zeigen sich jedoch deutliche Unterschiede in den Einschlusskriterien der beiden Studien. Während in der D-CARE-Studie prä- und postmenopausale Frauen mit hohem Rezidivrisiko eingeschlossen wurden, wurden in der ABCSG-18-Studie lediglich postmenopausale Frauen mit niedrigem Rezidivrisiko eingeschlossen. Des Weiteren erfolgte in D-CARE eine dosisdichte Applikation von $120 \mathrm{mg}$ Dmab, während in der ABCSG-18-Studie lediglich 60 mg alle 6 Monate zum Einsatz kamen. Beide Studien haben relevante Protokollveränderungen erfahren - aufgrund der niedrigen Rezidivrate in der D-CARE-Studie sowie der unerwartet hohen Fraktursenkung in der ABCSG-18Studie. Trotz all der oben beschriebenen studienspezifischen Unterschiede ist die deutliche Diskrepanz der Studienergebnisse nach wie vor überraschend und nicht zu erklären. Auf der Basis der D-CARE-Studie kann der adjuvante Einsatz von 120 mg Dmab, insbesondere aufgrund des hohen Nebenwirkungsspektrums, derzeit nicht empfohlen werden. Bei postmenopausalen Frauen unter Al-Therapie ist der frakturreduzierende Effekt von $60 \mathrm{mg}$ Dmab alle 6 Monate unbestritten. Auf der Basis der ABCSG-18Studie kann hier auch von einem positiven Effekt auf das DFS ausgegangen werden.

\section{Das frühe Mammakarzinom - die Behandlung der HER2-positiven Patientin}

Das Überleben von Patientinnen mit frühem Mammakarzinom mit einer neoadjuvanten oder adjuvanten Chemotherapie ist vergleichbar [74]. Aber heute wird der neoadjuvanten Chemotherapie häufig der Vorzug gegeben, wenn eine klare Indikation für eine postoperative Chemotherapie gegeben ist. Es konnte in mehreren Studien insbesondere bei HER2-positiven und triple-negativen Tumoren gezeigt werden, dass die pathologische Komplettremission $(p C R)$ ein guter Surrogatmarker für die Prognose ist [75-77]. Die Wahl der optimalen neoadjuvanten Therapie bei HER2-positiven Tumoren ist derzeit Gegenstand intensiver klinischer Forschung. Nach den bahnbrechenden Ergebnissen mit Trastuzumab in der Adjuvanz und Neoadjuvanz ist die HER2-spezifische Therapie der Standard. Aber welches sind die optimalen Kombinationspartner? In der Neosphere-Studie zeigte die duale Blockade mit Trastuzumab und Pertuzumab in Kombination mit Chemotherapie eine signifikant höhere pCR im Vergleich zu Trastuzmab alleine [78]. Bei kleineren Tumoren, bei fehlendem Lymphknotenbefall und bei „triple-positiven Tumoren“ (ER+/PR+/HER2+) werden jedoch auch andere Therapiekonzepte im Sinne einer Deeskalation zur Verringerung der Toxizität diskutiert.

In der Phase-II-Studie PerELISA wurde eine Kombination von Trastuzumab, Lapatinib und Letrozol bei HER2-positiven Patientinnen in einem adaptiven Design geprüft [79]. Die Patientinnen erhielten zunächst eine 2-wöchige Therapie mit Lapatinib, anschließend erfolgte eine Re-Biopsie zur Evaluation von Ki-67. Patientinnen, die einen Abfall von Ki-67 von mehr als 20\% hatten, erhielten anschließend eine Kombination von Lapatinib, Trastuzumab und
Pertuzumab für 5 Zyklen. Patientinnen ohne Ki-67-Abfall wurden mit Trastuzumab, Pertuzumab und Paclitaxel behandelt. 44 von 64 Patientinnen (69\%) zeigten nach 2 Wochen ein Ki-67-Ansprechen und wurden nach Therapie mit Lapatinib, Trastuzumab und Pertuzumab operiert. Die pCR-Rate dieser Gruppe betrug 20,5\% (9 Patientinnen). Die pCR-Rate war signifikant höher in der intrinsischen Subtypgruppe „HER2 enriched“ als in anderen Subtypen $(45,5$ vs. $13,8 \%, p=0,042)$ [79]. Der intrinsische Subtyp korrelierte auch mit der Ki-67-Antwort. Somit erreichte die PerELISA-Studie das Haupt-Zielkriterium, in dem eine chemotherapiefreie Behandlung im adaptiven Design ein hohes Ansprechen zeigte.

Auf der Suche nach prädiktiven Biomarkern ist insbesondere der intrinsische „HER2 enriched“ (HER2-E) Subtyp im Fokus. In einer retrospektiven Analyse wurden diesbezüglich die beiden neoadjuvanten Studien PAMELA und TBCRC006 untersucht [80]. Alle Patientinnen erhielten eine neoadjuvante Therapie mit Lapatinib und Trastuzumab, hormonrezeptorpositive Patientinnen zusätzlich Letrozol oder Tamoxifen [80]. 65\% der Patientinnen waren in der HER2-E-Subgruppe, wobei die pCR in dieser Gruppe signifkant höher war im Vergleich zu den anderen Subgruppen $(35,1$ vs. 9,9\%). Eine positive Korrelation zur pCR zeigte sich außerdem in der HER2-high-Gruppe (36,1 vs. 8,2\%) [80]. Die Kombination beider Biomarker identifizierte nahezu $50 \%$ aller Patientinnen, die eine pCR mit einer zielgerichteten Therapie erreichten. Diese Biomarker müssen allerdings noch in prospektiv randomisierten Studien überprüft werden.

Während einerseits die APHINITY-Studie den Einsatz der dualen Therapie mit Pertuzumab und Trastuzumab bei Patientinnen mit einem HER2-positiven Mammakarzinom und hohem Risiko bestätigt hat [81], laufen weiterhin Bestrebungen, eine Deeskalation der Therapie zu erreichen. So wurde im Rahmen der APT-Studie der Einsatz von Trastuzumab mit einer 12-wöchigen Monotherapie mit Paclitaxel untersucht, und zeigte im nodal-negativen Kollektiv mit einer Tumorgröße unter $3 \mathrm{~cm}$ ein krankheitsfreies Überleben (DFS) von 93,3\% nach 7 Jahren $[82,83]$. Eine weitere wesentliche Fragestellung der Deeskalation wie auch des klinischen Alltags ist der Einsatz von Trastuzumab komplett ohne Chemotherapie - insbesondere bei älteren Patientinnen mit Komorbiditäten. Bisher lag hierzu keine ausreichende Datenlage vor. Im Rahmen der RESPECT-Studie wurden 275 70-80-jährige Patientinnen mit einem HER2-positiven Mammakarzinom ab einer Tumorgröße von 5 mm zur Therapie mit Trastuzumab mono oder in Kombination mit Chemotherapie nach Wahl des Arztes randomisiert [84]. Das mediane Alter war 73,5 Jahre. Das 3-Jahres-DFS lag bei $94,8 \%$ in der Kombinationsgruppe und $89,2 \%$ in der Monotherapiegruppe (HR 1,42 [95\%-KI 0,68-2,95]; $p=0,35)$. Die Autoren schlussfolgerten aber, dass die geringe Anzahl an Ereignissen (18 vs. 12) die exakte Evaluierung der Monotherapie deutlich einschränkt. Allerdings berechneten sie, dass durch den Verzicht auf eine Chemotherapie die Lebenserwartung nach 3 Jahren lediglich um einen Monat reduziert wird und dies somit eine adjuvante Option für die ältere Patientin darstellen kann [84].

Zahlreiche Studien untersuchten zudem die Option der verkürzten Trastuzumab-Therapie, um sowohl Toxizitäten als auch Kosten zu reduzieren, nachdem im Rahmen der HERA-Studie für eine 2-jährige Therapie mit Trastuzumab kein Benefit im Vergleich zur 1-jährigen Therapie gezeigt werden konnte [85]. Bisherige Stu- 


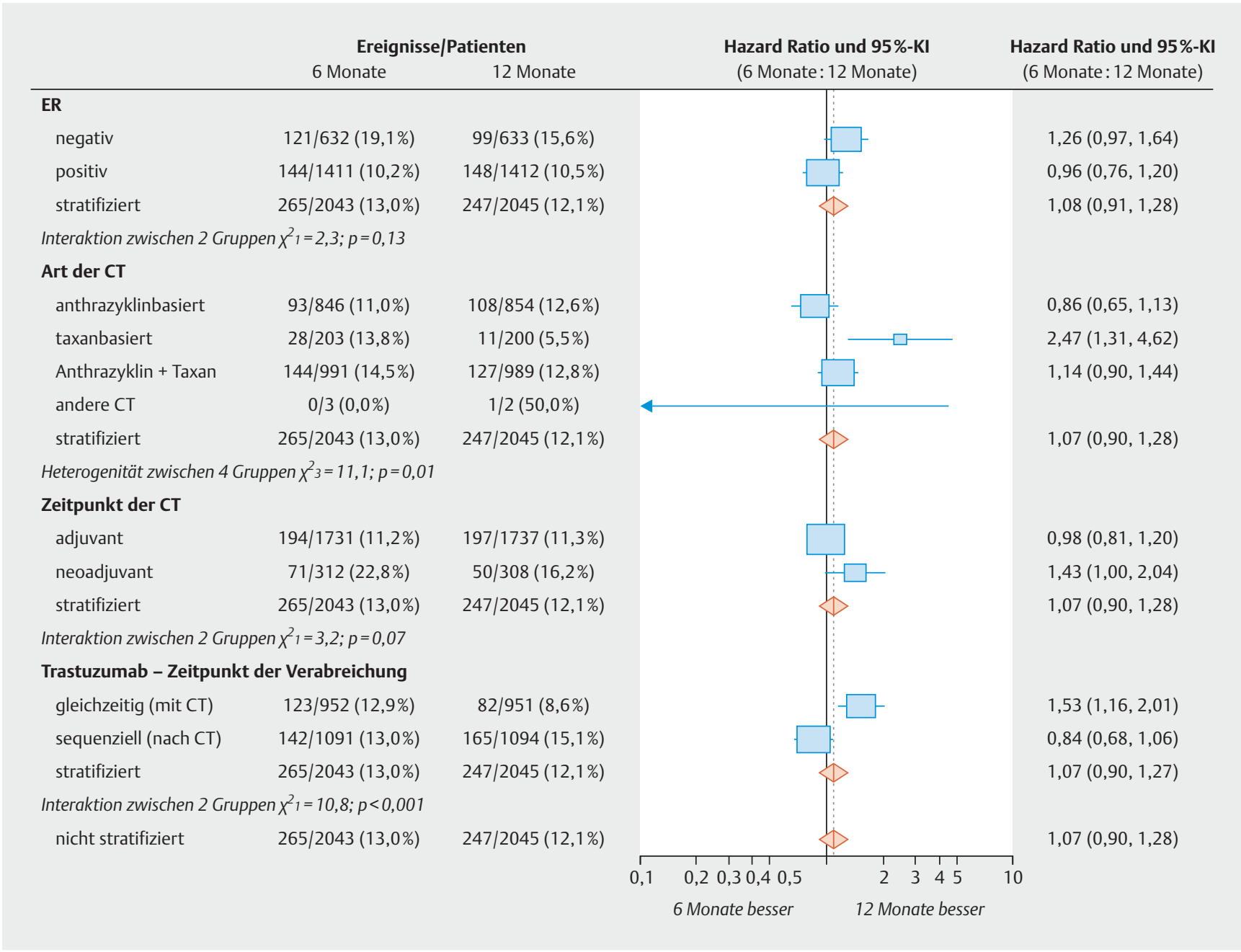

- Abb. 1 Geplante Subgruppenanalysen im Rahmen der PERSEPHONE-Studie in Bezug auf das rückfallfreie Überleben (DFS), modifiziert nach [88].

dien, wie die SOLD-Studie ( 9 Wochen Trastuzumab), die PHAREStudie (6 Monate Trastuzumab) oder auch die ShortHer-Studie (9 Wochen Trastuzumab) konnten eine Nichtunterlegenheit der verkürzten Anti-HER2-Therapie nicht bestätigen $[86,87]$. Im Rahmen der multizentrischen Phase-III-Studie PERSEPHONE wurde die Nichtunterlegenheit einer 6-monatigen Therapie im Vergleich zu 12 Monaten an einem großen Kollektiv mit 4089 Patientinnen erneut untersucht [88]. Es konnten Patientinnen mit einem HER2positiven Mammakarzinom und Indikation zur Chemotherapie bis zum 10. Zyklus Trastuzumab eingeschlossen werden. Nach einem medianen Follow-up von 5,4 Jahren zeigte sich kein signifikanter Unterschied beim 4-Jahres-DFS (89,4 vs. 89,8\%, HR 1,07 [95\%-KI $0,93-1,24]$ ). Auch in Bezug auf das Gesamtüberleben konnte die Nichtunterlegenheit der verkürzten Therapie bestätigt werden (93,8 vs. 94,8\%, HR 1,14 [95\%-KI 0,95-1,37]) [88]. Zudem war ein vorzeitiges Therapieende aufgrund einer Kardiotoxizität signifikant reduziert (4 vs. $8 \%, p<0,0001$ ). In den Subgruppenanalysen zeigten sich allerdings Kollektive, die von der 12-monatigen Therapie profitieren, nämlich östrogenrezeptornegative Patientinnen, solche, die eine reine taxanhaltige Chemotherapie erhielten, Patientinnen, die mit einer neoadjuvanten Therapie behandelt wurden, und Patientinnen mit synchroner Trastuzumab-Gabe ( $\bullet$ Abb. 1).

\section{Prognose- und Prädiktivmarker}

Die Verbesserung der Vorhersage der Prognose von Mammakarzinompatientinnen hat die Wissenschaft schon seit einiger Zeit beschäftigt. Neben der Verbesserung der Prognoseprädiktion durch Prädiktionsmodelle $[89,90]$ war insbesondere die Einführung von Multigentests einer der großen Fortschritte der letzen beiden Jahrzehnte [91-98].

Kürzlich konnten Langzeitdaten zum prognostischen und prädiktiven Wert eines 21-Gen-Expressionsassays (Oncotype DX Recurrence Sore) beim hormonrezeptorpositiven, HER2-negativen, nodal-negativen Mammakarzinom wurden im Rahmen der TAILOR-X-Studie präsentiert werden [93]. Bislang war aus früheren Analysen bekannt, dass das Testverfahren den prognostischen Wert für das Rückfallrisiko in einem Niedrigrisikokollektiv (Recurrence Score/RS 0-10) bei alleiniger endokriner Therapie sowie den prädiktiven Wert bei Patientinnen mit einem hohen Risiko (größer/gleich 26) nach einer Chemotherapie abbilden kann [94]. 
In der prospektiv-randomisierten Studie wurden aktuell die Daten für das mittlere Risikokollektiv vorgestellt (RS 11-25): Im randomisierten Vergleich erfolgte die Applikation einer endokrinen vs. chemoendokrinen Therapie bei diesen Patientinnen (zwischen 18 und 75 Lebensjahren). Der primäre Endpunkt wurde als Invasive Disease-Free Survival (iDFS) definiert, die Studie war konzipiert, um eine potenzielle Nichtunterlegenheit einer alleinigen endokrinen Therapie aufzuzeigen. Insgesamt konnten die Datensätze von 10253 Patientinnen ausgewertet werden. Von diesen zeigten 6711 (65,5\%) einen RS zwischen 11 und 25. Es zeigten sich 836 DFS-Events nach einem Follow-up von 90 Monaten. In der Gruppe der alleinig endokrin therapierten Patientinnen betrug das iDFS 83,3\% und in der Gruppe der sequenziell chemoendokrin therapierten Patientinnen 84,3\%: Die Gabe einer alleinigen endokrinen Therapie war in den Analysen und in der Intentionto-treat-(ITT-)Gruppe gegenüber der kombinierten Applikation einer chemo- und endokrinen Therapie hinsichtlich des iDFS somit nicht unterlegen (HR 1,08, 95\%-KI 0,94, 1,24, p=0,26) [93]. Die Gabe einer alleinigen endokrinen Therapie war ebenfalls nicht unterlegen für weitere Endpunkte wie Distant Recurrence-Free Interval (DRFI; HR 1,03, $\mathrm{p}=0,80$ ), Recurrence-Free-Interval (RFI; HR 1,12; $p=0,28$ ) und das Gesamtüberleben (OS, HR 0,97, $\mathrm{p}=0,80$ ) bei Patientinnen mit einem Alter über 50 und einem RS von 11-25 und bei Patientinnen jünger als 50 mit einem RS von 11-15. Auch wenn dieser randomisierte Vergleich durch die Darstellung der Langzeitdaten der Intermediärgruppe Hinweise auf die Planung einer endokrinen oder chemoendokrinen Therapie gibt, sollten in der klinischen Routine zunächst die klassischen (pathologischen) prognostischen und prädiktiven Faktoren für die Therapieplanung im Vordergrund stehen.

Danksagung

Diese Arbeit entstand teilweise in Folge von Förderungen der Firma Hexal und des PRAEGNANT Netzwerks, die keinen Anteil bei der Verfassung dieses Manuskriptes hatten. Für den Inhalt des Manuskriptes sind alleine die Autoren verantwortlich.

\section{Interessenkonflikt}

A. D. H. erhielt Honorare von AstraZeneca, Genomic Health, Roche, Novartis, Celgene und Pfizer. N. N. erhielt Beratungshonorare von Janssen-Cilag und Reisekostenzuschüsse von Novartis. F. O. erhielt Sprecherund Beratungshonorare von Amgen, Celgene, TEVA, AstraZeneca, Novartis, Roche und MSD. F.-A.T. erhielt Honorare von AstraZeneca, Genomic Health und Novartis. H.-C. K. erhielt Honorare von Carl Zeiss meditec, TEVA, Theraclion, Novartis, Amgen, AstraZeneca, Pfizer, Janssen-Cilag, GSK, LIV Pharma, Roche und Genomic Health. P. H. erhielt Honorare, unbeschränkte Fortbildungszuschüsse und Forschungsgelder von Amgen, AstraZeneca, Eli Lilly, MSD, Novartis, Pfizer und Roche. P. A. F. erhielt Honorare von Roche, Pfizer, Novartis und Celgene. Seine Einrichtung führt Forschungsprojekte für Novartis durch. H. T. erhielt Honorare von Novartis, Roche, Celgene, TEVA, Pfizer und Reisekostenzuschüsse von Roche, Celgene und Pfizer. J. E. erhielt Honorare von Roche, Celgene, Novartis, Pfizer, Pierre Fabre und TEVA sowie Reisekostenzuschüsse von Celgene, Pfizer, TEVA und Pierre Fabre. M. P. L. war in Beratungsausschüssen für AstraZeneca, MSD, Novartis, Pfizer, Genomic Health und Roche tätig und hat Vortragshonorare von MSD, Lilly, Roche, Novartis, Pfizer, Genomic Health, AstraZeneca, medac und Eisai erhalten. V. M. erhielt Sprecherhonorare von Amgen, AstraZeneca, Celgene, Daiichi-Sankyo, Eisai, Pfizer, Pierre-Fabre, Novartis, Roche, Teva und
Janssen-Cilag sowie Beratungshonorare von Genomic Health, Roche, Pierre Fabre, Amgen, Daiichi-Sankyo und Eisai. E. B. erhielt Honorare von Novartis und Hexal für Beratertätigkeiten und Managementfunktionen in der klinischen Forschung. A. S. erhielt Honorare von Roche, Celgene, AstraZeneca, Novartis, Pfizer, Zuckschwerdt Verlag GmbH, Georg Thieme Verlag, Aurikamed GmbH, MCl Deutschland GmbH, bsh medical communications $\mathrm{GmbH}$ und promedicis $\mathrm{GmbH}$. W. J. erhielt Honorare und Forschungsförderungen von Novartis, Roche, Pfizer, Lilly, AstraZeneca, Chugai, Sanofi, Daiichi Sankyo und Tesaro. F. S. war in Beratungsausschüssen für Novartis, Lilly, Amgen und Roche tätig und erhielt Vortragshonorare von Roche, AstraZeneca, MSD, Novartis und Pfizer. A. W. war in Beratungsausschüssen für Novartis, Amgen, Pfizer, Roche, Tesaro, Eisai tätig und erhielt Vortragshonorare von Novartis, Pfizer, Aurikamed, Roche, Celgene. D. L. erhielt Honorare von Amgen,

AstraZeneca, Celgene, Lilly, Loreal, MSD, Novartis, Pfizer, Tesaro. M. W. erhielt Sprecher- und Beratungshonorare von Novartis, Amgen, Celgene, Roche, Genentech, AstraZeneca und Pfizer. S. Y. B. erhielt Honorare von Pfizer und Novartis. T. N. F. war in Beratungsausschüssen für Amgen, Daiichi Sankyo, Novartis, Pfizer und Roche tätig und erhielt Vortragshonorare von Celgene, Roche, Novartis und Pfizer.

\section{Literatur}

[1] Taran FA, Schneeweiss A, Lux MP et al. Update Breast Cancer 2018 (Part 1) - Primary Breast Cancer and Biomarkers. Geburtsh Frauenheilk 2018; 78: $237-245$

[2] Schneeweiss A, Lux MP, Janni W et al. Update Breast Cancer 2018 (Part 2) - Advanced Breast Cancer, Quality of Life and Prevention. Geburtsh Frauenheilk 2018; 78: 246-259

[3] Untch M, Wurstlein R, Marschner $\mathrm{N}$ et al. 4th International Consensus Conference on Advanced Breast Cancer (ABC4), Lisbon, November 4, 2017: ABC4 Consensus: Assessment by a Panel of German Experts. Geburtsh Frauenheilk 2018; 78: 469-480

[4] Ghoussaini M, Fletcher O, Michailidou K et al. Genome-wide association analysis identifies three new breast cancer susceptibility loci. Nat Genet 2012; 44: 312-318

[5] Michailidou K, Beesley J, Lindstrom S et al. Genome-wide association analysis of more than 120,000 individuals identifies 15 new susceptibility loci for breast cancer. Nat Genet 2015; 47: 373-380

[6] Michailidou K, Hall P, Gonzalez-Neira A et al. Large-scale genotyping identifies 41 new loci associated with breast cancer risk. Nat Genet 2013; 45: 353-361, 361.e1-361.e2

[7] Michailidou K, Lindstrom S, Dennis J et al. Association analysis identifies 65 new breast cancer risk loci. Nature 2017; 551: 92-94

[8] Ahmed S, Thomas G, Ghoussaini M et al. Newly discovered breast cancer susceptibility loci on 3p24 and 17q23.2. Nat Genet 2009; 41: 585-590

[9] Antoniou AC, Wang X, Fredericksen ZS et al. A locus on 19p13 modifies risk of breast cancer in BRCA1 mutation carriers and is associated with hormone receptor-negative breast cancer in the general population. Nat Genet 2010; 42: 885-892

[10] Bojesen SE, Pooley KA, Johnatty SE et al. Multiple independent variants at the TERT locus are associated with telomere length and risks of breast and ovarian cancer. Nat Genet 2013; 45: 371-384, 384e1-384e2

[11] Cox A, Dunning AM, Garcia-Closas $M$ et al. A common coding variant in CASP8 is associated with breast cancer risk. Nat Genet 2007; 39: 352358

[12] Easton DF, Pooley KA, Dunning AM et al. Genome-wide association study identifies novel breast cancer susceptibility loci. Nature 2007; 447: 1087-1093

[13] Fasching PA, Ekici AB, Adamietz BR et al. Breast Cancer Risk - Genes, Environment and Clinics. Geburtsh Frauenheilk 2011; 71: 1056-1066

[14] Fasching PA, Ekici AB, Wachter DL et al. Breast Cancer Risk - From Genetics to Molecular Understanding of Pathogenesis. Geburtsh Frauenheilk 2013; 73: 1228-1235 
[15] Fletcher O, Johnson N, Orr N et al. Novel breast cancer susceptibility locus at 9q31.2: results of a genome-wide association study. J Natl Cancer Inst 2011; 103: 425-435

[16] French JD, Ghoussaini M, Edwards SL et al. Functional variants at the $11 q 13$ risk locus for breast cancer regulate cyclin D1 expression through long-range enhancers. Am J Hum Genet 2013; 92: 489-503

[17] Garcia-Closas M, Couch FJ, Lindstrom S et al. Genome-wide association studies identify four ER negative-specific breast cancer risk loci. Nat Genet 2013; 45: 392-398, 398e1-398e2

[18] Haiman CA, Chen GK, Vachon CM et al. A common variant at the TERTCLPTM1 L locus is associated with estrogen receptor-negative breast cancer. Nat Genet 2011; 43: 1210-1214

[19] Milne RL, Benitez J, Nevanlinna $\mathrm{H}$ et al. Risk of estrogen receptor-positive and -negative breast cancer and single-nucleotide polymorphism 2q35rs13387042. J Natl Cancer Inst 2009; 101: 1012-1018

[20] Milne RL, Kuchenbaecker KB, Michailidou K et al. Identification of ten variants associated with risk of estrogen-receptor-negative breast cancer. Nat Genet 2017; 49: 1767-1778

[21] Siddiq A, Couch FJ, Chen GK et al. A meta-analysis of genome-wide association studies of breast cancer identifies two novel susceptibility loci at 6q14 and 20q11. Hum Mol Genet 2012; 21: 5373-5384

[22] Stacey SN, Manolescu A, Sulem P et al. Common variants on chromosomes 2 q35 and $16 q 12$ confer susceptibility to estrogen receptor-positive breast cancer. Nat Genet 2007; 39: 865-869

[23] Stacey SN, Manolescu A, Sulem P et al. Common variants on chromosome 5 p12 confer susceptibility to estrogen receptor-positive breast cancer. Nat Genet 2008; 40: 703-706

[24] Stevens KN, Fredericksen Z, Vachon CM et al. 19p13.1 is a triple-negative-specific breast cancer susceptibility locus. Cancer Res 2012; 72 1795-1803

[25] Thomas G, Jacobs KB, Kraft P et al. A multistage genome-wide association study in breast cancer identifies two new risk alleles at 1p11.2 and 14q24.1 (RAD51L1). Nat Genet 2009; 41: 579-584

[26] Turnbull C, Ahmed S, Morrison J et al. Genome-wide association study identifies five new breast cancer susceptibility loci. Nat Genet 2010; 42: 504-507

[27] Wunderle M, Olmes G, Nabieva N et al. Risk, Prediction and Prevention of Hereditary Breast Cancer - Large-Scale Genomic Studies in Times of Big and Smart Data. Geburtsh Frauenheilk 2018; 78: 481-492

[28] Zheng W, Long J, Gao YT et al. Genome-wide association study identifies a new breast cancer susceptibility locus at 6q25.1. Nat Genet 2009; 41: 324-328

[29] Purrington KS, Slager S, Eccles D et al. Genome-wide association study identifies 25 known breast cancer susceptibility loci as risk factors for triple-negative breast cancer. Carcinogenesis 2014; 35: 1012-1019

[30] Stevens KN, Fredericksen Z, Vachon CM et al. 19p13.1 is a triple-negative-specific breast cancer susceptibility locus. Cancer Res 2012; 72: 1795-1803

[31] Vachon CM, Scott CG, Fasching PA et al. Common breast cancer susceptibility variants in LSP1 and RAD51L1 are associated with mammographic density measures that predict breast cancer risk. Cancer Epidemiol Biomarkers Prev 2012; 21: 1156-1166

[32] Garcia-Closas M, Gunsoy NB, Chatterjee N. Combined associations of genetic and environmental risk factors: implications for prevention of breast cancer. J Natl Cancer Inst 2014; 106: pii: dju305. doi:10.1093/ jnci/dju305

[33] Mavaddat N, Pharoah PD, Michailidou K et al. Prediction of breast cancer risk based on profiling with common genetic variants. J Natl Cancer Inst 2015; 107: pii: djv036. doi:10.1093/jnci/djv036

[34] Vachon CM, Pankratz VS, Scott CG et al. The contributions of breast density and common genetic variation to breast cancer risk. J Natl Cancer Inst 2015; 107: pii: dju397. doi:10.1093/jnci/dju397
[35] Rudolph A, Song M, Brook MN et al. Joint associations of a polygenic risk score and environmental risk factors for breast cancer in the Breast Cancer Association Consortium. Int J Epidemiol 2018. doi:10.1093/ije/ dyx 242

[36] Haberle L, Hein A, Rubner M et al. Predicting Triple-Negative Breast Cancer Subtype Using Multiple Single Nucleotide Polymorphisms for Breast Cancer Risk and Several Variable Selection Methods. Geburtsh Frauenheilk 2017; 77: 667-678

[37] Simard ], Chiarelli AM. Personalized risk assessment for prevention and early detection of breast cancer: Integration and Implementation. 2018. Online: https:/www.genomecanada.ca/sites/default/files/ 2017/sarp_backgrounder_en.pdf; Stand: 01.04.2018

[38] Unicancer. Randomized, Comparison Of Risk-Stratified versus Standard Breast Cancer Screening In European Women Aged 40-74. 2017. Online: https://cordis.europa.eu/project/rcn/212694_en.html; Stand: 01.04.2018

[39] Shieh Y, Eklund M, Madlensky L et al. Breast Cancer Screening in the Precision Medicine Era: Risk-Based Screening in a Population-Based Trial. J Natl Cancer Inst 2017. doi:10.1093/jnci/djw290

[40] Evans DG, Astley S, Stavrinos $\mathrm{P}$ et al. Improvement in risk prediction, early detection and prevention of breast cancer in the NHS Breast Screening Programme and family history clinics: a dual cohort study. Southampton (UK): NIHR Journals Library; 2016

[41] ASSURE Consortium. Final Report Summary - ASSURE (Adapting Breast Cancer Screening Strategy Using Personalised RiskEstimation). 2016. Online: https://cordis.europa.eu/result/rcn/187468_en.html; Stand: 01.04.2018

[42] Couch FJ, Hart SN, Sharma P et al. Inherited mutations in 17 breast cancer susceptibility genes among a large triple-negative breast cancer cohort unselected for family history of breast cancer. J Clin Oncol 2015; 33 : 304-311

[43] Couch FJ, Shimelis H, Hu C et al. Associations Between Cancer Predisposition Testing Panel Genes and Breast Cancer. JAMA Oncol 2017; 3: 1190-1196

[44] Fasching PA, Loibl S, Hu C et al. BRCA1/2 Mutations and Bevacizumab in the Neoadjuvant Treatment of Breast Cancer: Response and Prognosis Results in Patients With Triple-Negative Breast Cancer From the GeparQuinto Study. J Clin Oncol 2018. doi:10.1200/JCO.2017.77.2285

[45] Wunderle M, Gass P, Haberle L et al. BRCA mutations and their influence on pathological complete response and prognosis in a clinical cohort of neoadjuvantly treated breast cancer patients. Breast Cancer Res Treat 2018; 171: 85-94

[46] Fasching PA, Hu C, Hart SN et al. Cancer predisposition genes in metastatic breast cancer - Association with metastatic pattern, prognosis, patient and tumor characteristics [abstract]. In: Proceedings of the 2017 San Antonio Breast Cancer Symposium; 2017 Dec 5-9; San Antonio, TX. Philadelphia (PA): AACR; Cancer Res 2018; 78: Abstr. PD1-02

[47] Nolan E, Vaillant F, Branstetter D et al. RANK ligand as a potential target for breast cancer prevention in BRCA1-mutation carriers. Nat Med 2016; 22: 933-939

[48] Sigl V, Jones LP, Penninger JM. RANKL/RANK: from bone loss to the prevention of breast cancer. Open Biol 2016; 6: pii: 160230

[49] Sigl V, Owusu-Boaitey K, Joshi PA et al. RANKL/RANK control Brca1 mutation-driven mammary tumors. Cell Res 2016; 26: 761-774

[50] Bayer CM, Beckmann MW, Fasching PA. Updates on the role of receptor activator of nuclear factor kappaB/receptor activator of nuclear factor kappaB ligand/osteoprotegerin pathway in breast cancer risk and treatment. Curr Opin Obstet Gynecol 2017; 29: 4-11

[51] Francis P, Singer C, Saunders C et al. BRCA-P: An international randomised phase III study evaluating the RANK ligand inhibitor denosumab for the prevention of breast cancer in BRCA1 mutation carriers [20182022]. Online: http://purlorg/au-research/grants/nhmrc/1140715 and https://researchdataandsorgau/brca-p-an-mutation-carriers/1319302 2018; Stand: 02.04.2018 
[52] Kuchenbaecker KB, Hopper JL, Barnes DR et al. Risks of Breast, Ovarian, and Contralateral Breast Cancer for BRCA1 and BRCA2 Mutation Carriers. JAMA 2017; 317: 2402-2416

[53] Copson ER, Maishman TC, Tapper W] et al. Germline BRCA mutation and outcome in young-onset breast cancer (POSH): a prospective cohort study. Lancet Oncol 2018. doi:10.1016/S1470-2045(17)30891-4

[54] Fasching PA. Breast cancer in young women: do BRCA1 or BRCA2 mutations matter? Lancet Oncol 2018. doi:10.1016/S1470-2045(18)30008-1

[55] Evron E, Ben David MA, Goldberg $\mathrm{H}$ et al. Phase II national clinical trial of prophylactic irradiation to the contralateral breast for BRCA mutation carriers treated for early breast cancer (EBC). J Clin Oncol 2018; 36 (Suppl.): Abstr. 514

[56] Poortmans PM, Collette S, Kirkove C et al. Internal Mammary and Medial Supraclavicular Irradiation in Breast Cancer. N Engl J Med 2015; 373 : 317-327

[57] Poortmans P, Collette S, Struikmans H et al. Fifteen-year results of the randomised EORTC trial 22922/10925 investigating internal mammary and medial supraclavicular (IM-MS) lymph node irradiation in stage I-III breast cancer. J Clin Oncol 2018; 36 (Suppl.): Abstr. 504

[58] Herrera FG, Bourhis ], Coukos G. Radiotherapy combination opportunities leveraging immunity for the next oncology practice. CA Cancer J Clin 2017; 67: 65-85

[59] McArthur HL, Barker CA, Gucalp A et al. A phase II, single arm study assessing the efficacy of pembrolizumab (Pembro) plus radiotherapy (RT) in metastatic triple negative breast cancer (mTNBC). J Clin Oncol 2018; 36 (Suppl.): Abstr. 1017

[60] Formenti SC, Golden EB, Goldberg JD et al. Results of a phase I-II study of adjuvant concurrent carboplatin and accelerated radiotherapy for triple negative breast cancer. Oncoimmunology 2017; 6: e1274479

[61] Ngwa W, Irabor OC, Schoenfeld JD et al. Using immunotherapy to boost the abscopal effect. Nat Rev Cancer 2018; 18: 313-322

[62] Davies C, Godwin J, Gray R et al.; Early Breast Cancer Trialists' Collaborative Group. Relevance of breast cancer hormone receptors and other factors to the efficacy of adjuvant tamoxifen: patient-level meta-analysis of randomised trials. Lancet 2011; 378: 771-784

[63] Early Breast Cancer Trialists' Collaborative Group. Aromatase inhibitors versus tamoxifen in early breast cancer: patient-level meta-analysis of the randomised trials. Lancet 2015; 386: 1341-1352

[64] Pagani O, Regan MM, Walley BA et al. Adjuvant exemestane with ovarian suppression in premenopausal breast cancer. N Engl J Med 2014; 371: 107-118

[65] Francis PA, Regan MM, Fleming GF et al. Adjuvant ovarian suppression in premenopausal breast cancer. N Engl J Med 2015; 372: 436-446

[66] Francis PA, Pagani O, Fleming GF et al. Tailoring Adjuvant Endocrine Therapy for Premenopausal Breast Cancer. N Engl J Med 2018; 379: 122-137

[67] Regan MM, Francis PA, Pagani $O$ et al. Absolute improvements in freedom from distant recurrence with adjuvant endocrine therapies for premenopausal women with hormone receptor-positive $(\mathrm{HR}+)$ HER2-negative breast cancer (BC): Results from TEXT and SOFT. J Clin Oncol 2018; 36 (Suppl.): Abstr. 503

[68] Noh WC, Lee JW, Nam S] et al. Role of adding ovarian function suppression to tamoxifen in young women with hormone-sensitive breast cancer who remain premenopausal or resume menstruation after chemotherapy: The ASTRRA study. J Clin Oncol 2018; 36 (Suppl.): Abstr. 502

[69] Leitlinienprogramm Onkologie (Deutsche Krebsgesellschaft und Deutsche Krebshilfe und AWMF). S3-Leitlinie Früherkennung, Diagnose, Therapie und Nachsorge des Mammakarzinoms, Version 4.0, 2017 AWMF Registernummer: 032-045OL. 2017. Online: http://www.leitlinienprogrammonkologie.de/leitlinien/mammakarzinom/; Stand: 23.07.2018

[70] Early Breast Cancer Trialists' Collaborative Group. Adjuvant bisphosphonate treatment in early breast cancer: meta-analyses of individual patient data from randomised trials. Lancet 2015; 386: 1353-1361
[71] Gnant M, Pfeiler G, Dubsky PC et al. The impact of adjuvant denosumab on disease-free survival: Results from 3,425 postmenopausal patients of the ABCSG-18 trial. Cancer Res 2016; 76 (Suppl. 4): Abstract S2-02

[72] Coleman RE, Finkelstein D, Barrios CH et al. Adjuvant denosumab in early breast cancer: First results from the international multicenter randomized phase III placebo controlled D-CARE study. J Clin Oncol 2018; 36 (Suppl.): Abstr. 501

[73] Gnant M, Pfeiler G, Steger GG et al. Adjuvant denosumab in early breast cancer: Disease-free survival analysis of 3,425 postmenopausal patients in the ABCSG-18 trial. J Clin Oncol 2018; 36 (Suppl.): Abstr. 500

[74] Early Breast Cancer Trialists' Collaborative Group. Long-term outcomes for neoadjuvant versus adjuvant chemotherapy in early breast cancer: meta-analysis of individual patient data from ten randomised trials. Lancet Oncol 2018; 19: 27-39

[75] Cortazar P, Zhang L, Untch M et al. Pathological complete response and long-term clinical benefit in breast cancer: the CTNeoBC pooled analysis. Lancet 2014; 384: 164-172

[76] Fasching PA, Heusinger K, Haeberle L et al. Ki67, chemotherapy response, and prognosis in breast cancer patients receiving neoadjuvant treatment. BMC Cancer 2011; 11: 486

[77] von Minckwitz G, Untch M, Blohmer JU et al. Definition and impact of pathologic complete response on prognosis after neoadjuvant chemotherapy in various intrinsic breast cancer subtypes. J Clin Oncol 2012; 30: $1796-1804$

[78] Gianni L, Pienkowski T, Im YH et al. 5-year analysis of neoadjuvant pertuzumab and trastuzumab in patients with locally advanced, inflammatory, or early-stage HER2-positive breast cancer (NeoSphere): a multicentre, open-label, phase 2 randomised trial. Lancet Oncol 2016; 17 : 791-800

[79] Guarneri V, Dieci MV, Bisagni G et al. De-escalated treatment with trastuzumab-pertuzumab-letrozole in patients with $\mathrm{HR}+/ \mathrm{HER} 2+$ operable breast cancer with Ki67 response after 2 weeks letrozole: Final results of the PerELISA neoadjuvant study. ] Clin Oncol 2018; 36 (Suppl.): Abstr. 507

[80] Prat A, De Angelis C, Pascual T et al. HER2-enriched subtype and ERBB2 mRNA as predictors of pathological complete response following trastuzumab and lapatinib without chemotherapy in early-stage HER2positive breast cancer: A combined analysis of TBCRC006/023 and PAMELA trials. J Clin Oncol 2018; 36 (Suppl.): Abstr. 509

[81] von Minckwitz G, Procter M, de Azambuja E et al. Adjuvant Pertuzumab and Trastuzumab in Early HER2-Positive Breast Cancer. N Engl J Med 2017; 377: 122-131

[82] Tolaney SM, Barry WT, Dang CT et al. Adjuvant paclitaxel and trastuzumab for node-negative, HER2-positive breast cancer. N Engl J Med 2015; 372: 134-141

[83] Tolaney SM, Barry WT, Guo H et al. Seven-year (yr) follow-up of adjuvant paclitaxel (T) and trastuzumab (H) (APT trial) for node-negative, HER2positive breast cancer (BC). J Clin Oncol 2017; 35: 511

[84] Sawaki M, Saito T, Baba S et al. Evaluation of trastuzumab without chemotherapy as a postoperative adjuvant therapy in HER2-positive elderly breast cancer patients: Randomized controlled trial (RESPECT). J Clin Oncol 2018; 36 (Suppl.): Abstr. 510

[85] Cameron D, Piccart-Gebhart M], Gelber RD et al. 11 years' follow-up of trastuzumab after adjuvant chemotherapy in HER2-positive early breast cancer: final analysis of the HERceptin Adjuvant (HERA) trial. Lancet 2017; 389: 1195-1205

[86] Pivot X, Romieu G, Debled M et al. 6 months versus 12 months of adjuvant trastuzumab for patients with HER2-positive early breast cancer (PHARE): a randomised phase 3 trial. Lancet Oncol 2013; 14: 741-748

[87] Joensuu H, Fraser J, Wildiers $\mathrm{H}$ et al. Effect of Adjuvant Trastuzumab for a Duration of 9 Weeks vs. 1 Year With Concomitant Chemotherapy for Early Human Epidermal Growth Factor Receptor 2-Positive Breast Cancer: The SOLD Randomized Clinical Trial. JAMA Oncol 2018. doi:10.1001/jamaoncol.2018.1380 
[88] Earl HM, Hiller L, Vallier AL et al. PERSEPHONE: 6 versus 12 months (m) of adjuvant trastuzumab in patients (pts) with HER2 positive $(+)$ early breast cancer (EBC): Randomised phase 3 non-inferiority trial with definitive 4-year (yr) disease-free survival (DFS) results. J Clin Oncol 2018; 36 (Suppl.): Abstr. 506

[89] Ravdin PM, Siminoff LA, Davis G] et al. Computer program to assist in making decisions about adjuvant therapy for women with early breast cancer. J Clin Oncol 2001; 19: 980-991

[90] Wishart GC, Bajdik CD, Dicks E et al. PREDICT Plus: development and validation of a prognostic model for early breast cancer that includes HER2. Br J Cancer 2012; 107: 800-807

[91] Cardoso F, van't Veer L], Bogaerts J et al. 70-Gene Signature as an Aid to Treatment Decisions in Early-Stage Breast Cancer. N Engl J Med 2016; 375: 717-729

[92] Paik S, Shak S, Tang G et al. A multigene assay to predict recurrence of tamoxifen-treated, node-negative breast cancer. N Engl ] Med 2004; 351: $2817-2826$

[93] Sparano JA, Gray RJ, Makower DF et al. Adjuvant Chemotherapy Guided by a 21-Gene Expression Assay in Breast Cancer. N Engl J Med 2018; 379: $111-121$
[94] Sparano JA, Gray RJ, Makower DF et al. Prospective Validation of a 21 Gene Expression Assay in Breast Cancer. N Engl J Med 2015; 373: $2005-$ 2014

[95] Cuzick J, Dowsett M, Pineda S et al. Prognostic value of a combined estrogen receptor, progesterone receptor, Ki-67, and human epidermal growth factor receptor 2 immunohistochemical score and comparison with the Genomic Health recurrence score in early breast cancer. J Clin Oncol 2011; 29: 4273-4278

[96] Dowsett M, Sestak I, Lopez-Knowles E et al. Comparison of PAM50 risk of recurrence score with oncotype DX and IHC4 for predicting risk of distant recurrence after endocrine therapy. J Clin Oncol 2013; 31: 27832790

[97] Filipits M, Rudas M, Jakesz R et al. A new molecular predictor of distant recurrence in ER-positive, HER2-negative breast cancer adds independent information to conventional clinical risk factors. Clin Cancer Res 2011; 17: 6012-6020

[98] Schmidt M, Fasching PA, Beckmann MW et al. Biomarkers in Breast Cancer - An Update. Geburtsh Frauenheilk 2012; 72: 819-832 